DOI: https://doi.org/10.14311/TPFM.2022.003

\title{
THE RIGID BODY MOTION IN COSSERAT'S FLUID WITH NAVIER'S SLIP BOUNDARY CONDITIONS
}

\author{
Nikolai V. Chemetov \\ Department of Computing and Mathematics-FFCLRP, University of São Paulo, Av. Ban- \\ deirantes, 3900 - Monte Alegre, 14040-900 Ribeirão Preto, Brazil
}

\begin{abstract}
The aim of this article is to give a brief presentation of recent results related to the body-fluid interaction problem. The motion is described by a system of coupled differential equations: Newton's second law and Navier Stokes type equations. We shall formulate the global solvability result of weak solution of the problem, when the slippage is allowed at the boundaries of the rigid body and of the bounded domain, occupied by the fluid.
\end{abstract}

Keywords: rigid body, viscous fluid, collisions in finite time, global-in-time weak solution.

\section{Introduction}

The phenomenon of fluid-rigid interactions can be found in many branches of science, such as aeronautical, astronomical, marine, ocean, biological and medical engineering, etc. The mathematical study of fluid-rigid interaction problem, having a lot of applications, plays an important role in mathematics.

The motion of rigid bodies in a bounded domain, occupied by a viscous fluid, has been investigated by many authors, for example see $[4,12,13,14,19,22,25,27]$. These authors considered the non-slip boundary condition (classical Dirichlet's condition) at the boundaries of the body and of the domain. As well-known $[16,17,18,25]$, this assumption gave a paradoxical result, that during the motion of fluid and rigid body there can not happen collisions between the body and the domain boundary.

Various attempts have been made to resolve this paradox. One of more interesting, which was proposed in [21], is to permit the slippage of fluid particles on the boundaries of the body and of the domain. The slippage is prescribed by Navier's boundary conditions [6,7], having only the continuity of the velocity just in the normal component. More precisely, tangential components of the fluid velocity at the interface (between the rigid body and the fluid) need not be equal to tangential components of the velocity of the rigid body, implying a discontinuity of velocity at the boundary of the body. This fact significantly complicates studies of the problem.

Let us describe the model of the body-fluid motion with the assumption of slippage on the boundaries. We shall investigate the motion of a rigid body inside of a viscous fluid in a bounded 2-dimensional domain $\Omega$. We assume that at the initial moment $t=0$ the body occupies an open subset $S_{0}$ of $\Omega$. The motion of any material point $\mathbf{y}=\left(y_{1}, y_{2}\right)^{T} \in S_{0}$ on a time interval $[0, T]$ is described by an isometry

$$
\mathbf{A}(t, \mathbf{y})=\mathbf{q}(t)+\mathbb{Q}(t)(\mathbf{y}-\mathbf{q}(0)), \quad t \in[0, T],
$$

where $\mathbf{q}=\mathbf{q}(t) \in \mathbb{R}^{2}$ is the position of the body mass center and

$$
\mathbb{Q}(t)=\left[\begin{array}{cc}
\cos \theta(t) & -\sin \theta(t) \\
\sin \theta(t) & \cos \theta(t)
\end{array}\right]
$$

is the rotation matrix associated to the rotation angle $\theta=\theta(t) \in \mathbb{R}$ of the body. Therefore, the body occupies the set

$$
S(t)=\mathbf{A}\left(t, S_{0}\right)=\left\{\mathbf{x} \in \mathbb{R}^{2}: \mathbf{x}=\mathbf{A}(t, \mathbf{y}), \quad \mathbf{y} \in S_{0}\right\} \subset \Omega
$$

at any time moment $t \in[0, T]$. If we introduce the translation velocity $\mathbf{a}=\mathbf{a}(t) \in \mathbb{R}^{2}$ and the angular velocity $\omega=\omega(t) \in \mathbb{R}$ satisfying the equations

$$
\frac{d \mathbf{q}}{d t}=\mathbf{a}, \quad \frac{d \theta}{d t}=\omega \quad \text { in }[0, T],
$$

the body velocity $\mathbf{u}_{s}$ is calculated through the formula

$$
\mathbf{u}_{s}(t, \mathbf{x})=\mathbf{a}(t)+\omega(t)(\mathbf{x}-\mathbf{q}(t))^{\perp} \quad \text { for all } \quad \mathbf{x} \in S(t),
$$


where $\mathbf{x}^{\perp}=\left(-x_{2}, x_{1}\right)^{T}$ for any $\mathbf{x}=\left(x_{1}, x_{2}\right)^{T} \in \mathbb{R}^{2}$.

Assuming for simplicity of considerations that external forces are absent and the densities of the fluid and of the body are equal to the constant value 1, the model describing the fluid-body motion is the system of coupled differential equations, consisting from Newton's second law and Navier-Stokes type equations

$$
\begin{aligned}
m \frac{d \mathbf{a}}{d t} & =-\int_{\partial S(t)} \mathbb{T} \mathbf{n} d \gamma \quad \text { for } \mathbf{x} \in S(t), \\
\frac{d(I \omega)}{d t} & =-\int_{\partial S(t)}(\mathbf{x}-\mathbf{q}(t))^{\perp} \cdot \mathbb{T} \mathbf{n} d \gamma, \\
\partial_{t} \mathbf{u}_{f}+\left(\mathbf{u}_{f} \cdot \nabla\right) \mathbf{u}_{f} & =\operatorname{div} \mathbb{T}, \quad \operatorname{div} \mathbf{u}_{f}=0 \quad \text { for } \mathbf{x} \in F(t)=\Omega \backslash \overline{S(t)},
\end{aligned}
$$

where $d \gamma$ is the integration over the boundary $\partial S(t)$ of $S(t)$;

$$
m=\left|S_{0}\right| \quad \text { and } \quad I(t)=\int_{S(t)}|\mathbf{x}-\mathbf{q}(t)|^{2} d \mathbf{x}
$$

are the body mass and the inertia moment of the body $S(t)$, related to the mass center $\mathbf{q}=\mathbf{q}(t) ; \mathbf{n}=\mathbf{n}(\mathbf{x})-$ the unit interior normal at $\mathbf{x} \in \partial S(t) ; \mathbf{u}_{f}$ is the fluid velocity.

Many of fluids can not be described by the Newtonian constitutive relations, such as polymeric suspensions, slurries, animal blood, viscous polymers, etc.. Such fluids are characterized by symmetric and non-symmetric rate-of-strain tensors. For details we refer to the asymmetric continuum theory [23, 24, 26]. Let $|X|=\left(\sum_{i, j=1}^{2} x_{i j}^{2}\right)^{1 / 2}$ be the norm of a tensor $X \in \mathbb{R}^{2 \times 2}$. We assume that our fluid is described by the following Cauchy stress tensor

$$
\mathbb{T}=-p \mathrm{I}+\mathbb{S} \mathbf{u}_{f} \quad \text { with } \quad \mathbb{S} \mathbf{u}_{f}=2 \mu_{1}\left|\mathrm{~B}_{s}\right|^{p-2} \mathrm{~B}_{s}+\mu_{2}\left|\mathrm{~B}_{a}\right|^{p-2} \mathbf{B}_{a},
$$

where $p$ is the pressure and $\mu_{1}>0, \mu_{2}>0$ are constant viscosities;

$$
\mathrm{B}_{s} \mathbf{u}_{f}=\frac{\nabla \mathbf{u}_{f}+\left(\nabla \mathbf{u}_{f}\right)^{T}}{2} \quad \text { and } \quad \mathrm{B}_{s} \mathbf{u}_{f}=\frac{\nabla \mathbf{u}_{f}-\left(\nabla \mathbf{u}_{f}\right)^{T}}{2}
$$

are the symmetric and skew-symmetric parts of $\nabla \mathbf{u}_{f}$.

Let us define the common velocity and the common viscous part of the Cauchy stress tensor for the body-fluid medium

$$
\mathbf{u}=\left\{\begin{array}{ll}
\mathbf{u}_{s}, & \mathbf{x} \in S(t), \\
\mathbf{u}_{f}, & \mathbf{x} \in F(t),
\end{array} \quad \mathbb{S} \mathbf{u}=\left\{\begin{array}{ll}
\mathbf{0}, & \mathbf{x} \in S(t), \\
\mathbb{S u}_{f}, & \mathbf{x} \in F(t)
\end{array} \quad \text { in }(0, T) \times \Omega\right.\right.
$$

with $\mathbb{S} \mathbf{u}_{f}$ introduced in (6). We close the system (5) by the initial conditions

$$
S(0)=S_{0}, \quad \mathbf{u}(0)=\mathbf{u}_{0} \quad \text { at } t=0,
$$

and by Navier's boundary conditions

$$
\begin{aligned}
& \mathbf{u}_{f} \cdot \mathbf{n}=\mathbf{u}_{s} \cdot \mathbf{n}, \quad\left(\mathbb{T} \mathbf{n}+\alpha\left(\mathbf{u}_{f}-\mathbf{u}_{s}\right)\right) \cdot \boldsymbol{\tau}=0 \quad \text { on } \partial S(t), \\
& \mathbf{u} \cdot \mathbf{n}=0, \quad(\mathbb{T} \mathbf{n}+\beta \mathbf{u}) \cdot \boldsymbol{\tau}=0 \quad \text { on } \partial \Omega,
\end{aligned}
$$

prescribed on $\partial S(t)$ and on the boundary $\partial \Omega$ of $\Omega$. Here $\mathbf{n}, \boldsymbol{\tau}=\mathbf{n}^{\perp}$ are the external normal, tangent vector on $\partial S(t)$ and on $\partial \Omega$, respectively; the constants $\alpha, \beta>0$ are so-called friction coefficients.

Let us point that the existence of local-in-time solutions of the problem for different types of Newtonian fluids was shown in $[3,15,20]$. These existence results were obtained until a collision moment between the body and the wall of the domain. Also the local-in-time problem up to the collision time was studied in $[1,11]$, proving the existence and uniqueness of the weak-strong solutions.

The global-in-time solvability result of the problem was demonstrated in $[9,10]$. The obtained result is valid when Navier's conditions prescribed only at the body boundary with Dirichlet's zero condition on the domain boundary. Since the velocity was considered zero at the domain boundary, it was possible to extend the fluid domain to the whole space, obtaining that we can apply standard compactness techniques to approximate solutions in the extended fluid domain.

In the following section we announce the global solvability-in-time result for the posed problem (5)-(8). The result admits an arbitrary number of collisions between the body and the wall of the domain. 


\section{Main result}

Let us introduce some notations. For $\mathbf{v} \in \mathbb{R}^{2}$ we define the symmetric matrix $\mathbf{v} \otimes \mathbf{v}$ with the components $(\mathbf{v} \otimes \mathbf{v})_{i j}=u_{i} u_{j}$ for $i, j=1,2$ and introduce the divergence free space

$$
V^{0}(\Omega)=\left\{\mathbf{v} \in L^{2}(\Omega): \operatorname{div} \mathbf{v}=0 \text { in } \mathscr{D}^{\prime}(\Omega), \mathbf{v} \cdot \mathbf{n}=0 \text { in } H^{-1 / 2}(\partial \Omega)\right\},
$$

where we use standard notations of Lebesgue and Sobolev spaces and of the space of regular distributions over $\Omega$.

Let $S$ be an open subset of $\Omega$ with the Lipschitz boundary $\partial S \in C^{0,1}$. We also consider the following space of vector functions

$$
\begin{aligned}
K^{p}[\Omega, S]=\left\{\mathbf{v} \in V^{0}(\Omega): \quad\right. & \mathrm{B}_{S} \mathbf{v}, \mathrm{B}_{a} \mathbf{v} \in L^{p}(\Omega \backslash \bar{S}), \\
& \left.\mathrm{B}_{s} \mathbf{v}=0 \text { a.e. on } S, \quad[\mathbf{v}] \in L^{2}(\partial S)\right\},
\end{aligned}
$$

where $[\mathbf{v}]=\mathbf{v}_{s}-\mathbf{v}_{f}$ is the jump of the velocity on $\partial S$ and $\mathbf{v}_{s}, \mathbf{v}_{f}$ are the trace values of $\mathbf{v}$ on $\partial S$ from the "body" side $S$ and the "fluid" side $\Omega \backslash \bar{S}$, respectively.

Let us give the definition of the weak solutions of (5)-(8).

Definition 2.1 The pair $\{\mathbf{A}, \mathbf{u}\}$ is a weak solution of system (5)-(8), if the following three conditions are satisfied:

1) The function $\mathbf{A}(t, \cdot): \mathbb{R}^{2} \rightarrow \mathbb{R}^{2}$ is an isometry (1) defining a time dependent set $S(t)$, prescribed by (2). This isometry $\mathbf{A}$ and the velocity $\mathbf{u}=\mathbf{u}_{s}$ on $S(t)$, which is defined by (4), are related through of the equalities (1) and (3).

2) The functions $\mathbf{q}, \alpha$ are Lipschitz continuous on $[0, T]$;

3) The function

$$
\mathbf{u} \in L^{\infty}\left(0, T ; V^{0}(\Omega)\right) \cap L^{p}\left(0, T ; K^{p}[\Omega, S(t)]\right)
$$

satisfies the integral equality

$$
\begin{aligned}
\int_{0}^{T} \int_{\Omega \backslash \partial S(t)}\left\{\mathbf{u} \boldsymbol{\psi}_{t}\right. & +(\mathbf{u} \otimes \mathbf{u}-\mathbb{S u}): \nabla \boldsymbol{\psi}\} d \mathbf{x} d t+\int_{\Omega} \mathbf{u}_{0} \boldsymbol{\psi}(0) d \mathbf{x} \\
& =\int_{0}^{T}\left\{\alpha \int_{\partial S(t)}[\mathbf{u}][\boldsymbol{\psi}] d \gamma+\beta \int_{\partial \Omega} \mathbf{u} \boldsymbol{\psi} d \gamma\right\} d t
\end{aligned}
$$

which holds for any test function

$$
\begin{aligned}
\boldsymbol{\psi} \in \quad & K_{S, T}[\Omega]=\left\{\boldsymbol{\phi} \in L^{2}\left(0, T ; K^{2}[\Omega, S(t)]\right):\right. \\
& \left.\boldsymbol{\phi}_{t} \in L^{2}\left(0, T ; L^{2}(\Omega \backslash \partial S(t))\right) \quad \text { and } \quad \boldsymbol{\phi}(T, \cdot)=0 \quad \text { on } \Omega\right\},
\end{aligned}
$$

where we denote by

$$
[\mathbf{u}](t)=\left(\mathbf{u}_{s}-\mathbf{u}_{f}\right)(t), \quad[\boldsymbol{\psi}](t)=\left(\boldsymbol{\psi}_{s}-\boldsymbol{\psi}_{f}\right)(t)
$$

the jump of the trace values of $\mathbf{u}, \boldsymbol{\psi}$ on $\partial S(t)$.

Now we can state our main result.

Theorem 2.1 Let $S_{0}$ be a given open connected subset of a bounded domain $\Omega \subset \mathbb{R}^{2}$. We assume that $p \geqslant 4 / 3$,

$$
\begin{aligned}
& S_{0} \Subset \quad \Omega \text { and } \partial \Omega, \partial S_{0} \in C^{0,1}, \\
& \mathbf{u}_{0} \in V^{0}(\Omega), \quad \text { such that } \mathrm{B}_{s} \mathbf{u}_{0}=0 \quad \text { on } S_{0} .
\end{aligned}
$$

Then problem (5)-(8) has a weak solution $\{\mathbf{A}, \mathbf{u}\}$, satisfying for $t \in(0, T)$ the energy estimate

$$
\begin{aligned}
\frac{1}{2}\|\mathbf{u}(t)\|_{L^{2}(\Omega)}^{2} & +\int_{0}^{t}\left\{2 \mu_{1}\left\|\mathrm{~B}_{s} \mathbf{u}\right\|_{L^{p}(F(r))}^{p}+\mu_{2}\left\|\mathrm{~B}_{a} \mathbf{u}\right\|_{L^{p}(F(r))}^{p}\right. \\
& \left.+\left(\alpha\left\|\left.[\mathbf{u}]\right|_{L^{2}(\partial S(r))} ^{2}+\beta\right\| \mathbf{u} \|_{L^{2}(\partial \Omega)}^{2}\right)\right\} d r \leqslant \frac{1}{2}\left\|\mathbf{u}_{0}\right\|_{L^{2}(\Omega)}^{2} .
\end{aligned}
$$


In the proof of this theorem mathematical techniques, based on the previous works $[4,9,10,15]$, were developed to solve two principal difficulties, related with:

1) the definition of weak solution of problem (5)-(8): the solutions sought must belong to the $K^{p}[\Omega, S]$ space defined in (1), allowing jumps of the velocity field at the interface between the body and the fluid;

and

2) the lack of Sobolev-type embeeding theorems for the $K^{p}[\Omega, S]$-space. We refer to [8] (see also [2]), where the embedding theorem for the $K^{p}[\Omega, S]$-space was obtained in the domain with finite perimeter boundary.

For the details of the proof we refer to [5].

\section{Acknowledgment}

The author thanks Prof. Anna Mazzucato (Penn State University) and Prof. Šarka Nečasová (Institute of Mathematics, Prague, Czech Republic) for interesting discussions.

The work of N.V. Chemetov was supported by Fundação de Amparo à Pesquisa do Estado de São Paulo (FAPESP)Brasil, under the Project No.2021/03758-8: "Mathematical problems in fluid dynamics".

\section{References}

[1] Al Baba, H. \& Chemetov, N.V. \& Necasova, S. \& Muha, B.: Strong solutions in L2 framework for fluid-rigid body interaction problem-mixed case. Topological Methods in Nonlinear Analysis, vol. 52, no. 1: (2018) pp. 337-350.

[2] Arruda, L. \& Chemetov, N.V.: Embedding theorem for bounded deformations in domains with cusps. Math. Methods in the Applied Sciences, vol. 37, no. 17: (2014) pp. 2739-2745.

[3] Balilescu, L. \& San Martin, J. \& Takahashi, T.: Fluid-Rigid Structure Interaction System with Coulomb's Law. SIAM J. Math. Anal., vol. 49, no. 6: (2017) pp. 4625-4657.

[4] Bost, C. \& Cottet, G.-H. \& Maitre, E.: Convergence analysis of a penalization method for the three dimensional motion of a rigid body in an incompressible viscous fluid. SIAM J. Numer. Anal., vol. 48, no. 4: (2010) pp. 1313-1337.

[5] Chemetov, N.V.: Global solvability result for the rigid body motion in Cosserat's fluid with collisions.

[6] Chemetov, N.V. \& Antontsev, S.N.: Euler equations with non-homogeneous Navier slip boundary condition. Physica D: Nonlinear Phenomena, vol. 237: (2008) pp. 92-105.

[7] Chemetov, N.V. \& Cipriano, F.: Boundary layer problem: Navier-Stokes equations and Euler equations. Nonlinear Analysis: Real World Applications, vol. 14, no. 6: (2013) pp. 2091-2104.

[8] Chemetov, N.V. \& Mazzucato, A.: Embedding results in $\mathrm{LD}^{\wedge} \mathrm{p}$ on sets of finite perimeter. Proceedings of the Royal Society of Edinburgh, Section A: Mathematics, vol. 29: (2019) pp. 1-24.

[9] Chemetov, N.V. \& Nečasová, Š.: The motion of the rigid body in the viscous fluid including collisions. Global solvability result. Nonlinear Anal. Real World Appl., vol. 34: (2017) pp. 416-445.

[10] Chemetov, N.V. \& Nečasová, Š.: The Motion of a Rigid Body and a Viscous Fluid in a Bounded Domain in Presence of Collisions. Fluid Mechanics Research International Journal, vol. 2, no. 1: (2018) pp. 1-14.

[11] Chemetov, N.V. \& Nečasová, Š. \& Muha, B.: Weak-strong uniqueness for fluid-rigid body interaction problem with slip boundary condition. J. Math. Physics, vol. 60, no. 011505 (2019) pp. 1-13.

[12] Conca, C. \& San Martin, J. \& Tucsnak M.: Existence of solutions for the equations modelling the motion of a rigid body in a viscous fluid. Commun. Partial Differential Equation, vol. 25: (2000) pp. 1019-1042. 
[13] Feireisl, E.: On the motion of rigid bodies in a viscous compressible fluid. Arch. Rational Mech. Anal., vol. 167: (2003) pp. 281-308.

[14] Feireisl, E. \& Hillairet, M. \& Nečasová Š.: On the motion of several rigid bodies in an incompressible non-Newtonian fluid. Nonlinearity, vol. 21: (2008) pp. 1349-1366.

[15] Gérard-Varet, D. \& Hillairet, M.: Existence of weak solutions up to collision for viscous fluid-solid systems with slip. Comm. Pure Appl. Math., vol. 67, no. 12: (2014) pp. 2022-2075.

[16] Gérard-Varet, D. \& Hillairet, M. \& Wang, C.: The influence of boundary conditions on the contact problem in a 3D Navier-Stokes flow. J. Math. Pures Appl., vol. 9, no. 1: (2015) pp. 1-38.

[17] Hesla, T.I.: Collision of smooth bodies in a viscous fluid: A mathematical investigation. PhD Thesis - Minnesota (2005).

[18] Hillairet, M.: Lack of collision between solid bodies in a 2D incompressible viscous flow. Comm. Partial Differential Equations, vol. 32, no. 7-9: (2007) pp. 1345-1371.

[19] Hoffmann, K.-H. \& Starovoitov, V.N.: On a motion of a solid body in a viscous fluid. Two dimensional case. Adv. Math. Sci. Appl., vol. 9: (1999) pp. 633-648.

[20] Nečasová, Š. \& Ramaswamy, A.M. \& Roy, A. \& Schlomerkemper, A.: Motion of a rigid body in a compressible Fluid with Navier-slip boundary condition. ArXiv: 2103.08762 (2021).

[21] Neustupa, J. \& Penel P.: Existence of a weak solution to the Navier-Stokes equation with Navier's boundary condition around striking bodies. Comptes Rendus Mathematique, vol. 347, no. 11-12: (2009) pp. 685-690, .

[22] San Martin, J.A. \& Starovoitov, V.N. \& Tucsnak M.: Global weak solutions for the two dimensional motion of several rigid bodies in an incompressible viscous fluid. Arch. Rational Mech. Anal., vol. 161: (2002) pp. 93-112.

[23] Shelukhin, V.V. \& Chemetov N.V.: Global Solvability of the One-Dimensional Cosserat Bingham Fluid Equations. J. Math. Fluid Mech., vol. 17, no. 3: (2015) pp. 495-511.

[24] Shelukhin, V.V. \& Růžička M.: On Cosserat-Bingham Fluids. Z. Angew. Math. Mech., vol. 93, no. 1: (2013) pp. 57-72.

[25] Starovoitov, V.N.: Behavior of a rigid body in an incompressible viscous fluid near boundary. International Series of Numerical Mathematics, vol. 147: (2003) pp. 313-327.

[26] Teisseyre, R. \& Teisseyre-Jerenska M.: Asymmetric continuum: Extreme processes in solids and fluids. Springer, Heldelberg, New York, London (2014).

[27] Judakov, N.V.: The solvability of the problem of the motion of a rigid body in a viscous incompressible fluid. (Russian). Dinamika Splosnoi Sredy, vol. 18: (1974) pp. 249-253. 